\section{Estado nutricional e fatores associados à estatura de crianças da Terra Indígena Guarita, Sul do Brasil}

\author{
Nutritional status and social determinants \\ of child height in the Guarita Indigenous \\ Territory, Southern Brazil
}

\author{
Ivone Andreatta Menegolla 1 \\ Maria de Lourdes Drachler 2 \\ Inajara Haubert Rodrigues 3 \\ Lucio Roberto Schwingel 4 \\ Elaine Scapinello 5 \\ Maisa Beltrame Pedroso 5 \\ José Carlos de Carvalho Leite 2
}

\author{
1 Programa de Pós-graduação \\ em Ciências da Saúde \\ Universidade do Vale \\ do Rio dos Sinos, \\ São Leopoldo, Brasil. \\ 2 Institute of Health, \\ University of East Anglia, \\ Norwich, UK. \\ 3 Assessoria de Saúde \\ Indigena, Secretaria Estadual \\ da Saúde do Rio Grande \\ do Sul, Porto Alegre, Brasil. \\ ${ }^{4}$ Conselho de Missão entre \\ Indios, Secretaria Estadual \\ da Saúde do Rio Grande \\ do Sul, Porto Alegre, Brasil. \\ 5 Política de Alimentação \\ e Nutrição, Secretaria \\ Estadual da Saúde \\ do Rio Grande do Sul, \\ Porto Alegre, Brasil. \\ Correspondência \\ I. A. Menegolla \\ Programa de Pós-graduação \\ em Ciências da Saúde, \\ Universidade do Vale \\ do Rio dos Sinos. \\ Rua Costa Lima 790 \\ apto. 510, Porto Alegre, RS \\ 91720-480, Brasil. \\ ivonemenegolla@yahoo.com.br
}

\section{Abstract}

The nutritional status of under-five children and the association between social conditions and child stature were examined using data from the program to control malnutrition and mortality in the Guarita Indigenous Territory, southern Brazil, 2001-2002. Anthropometric indices were calculated in $z$-scores of the CDC 2000 reference. At entrance into the program, $34.7 \%$ of the children presented stunting, $12.9 \%$ low weight for age, $4.2 \%$ wasting, and $8.7 \%$ overweight. Stunting was most prevalent among boys and children older than one year. Multivariate linear regression showed that, on average, children were shorter when the drinking water was collected directly in the environment $(p=0.046)$, there was no refrigerator for food preservation ( $p=0.021)$, maternal age was less than 16 years at the birth of the oldest child among the under-fives ( $p=0.019$ ), and the mother was illiterate $(p=0.083)$. Sewage facilities only had an effect on the unadjusted model. There was no evidence that the number of under-five children had an effect on stature. Social inclusion policies and health and social provision which takes these factors into account are potentially relevant for improving health and nutrition in this population.

Nutritional Status; Social Inequity; South American Indians; Child

\section{Introdução}

Entre os indicadores nutricionais, os índices de altura para a idade são indicadores sensíveis dos processos de desnutrição ocorridos ao longo da vida da criança, refletindo condições de vida e saúde 1,2. Estudos da tendência secular do crescimento 3,4 e outros sobre determinantes de desigualdades em altura entre populações e diferentes grupos populacionais $5,6,7,8$ sugerem que, internacionalmente, os principais determinantes dos déficits de crescimento são as carências alimentares e doenças reincidentes 8,9 decorrentes de privações de condições elementares de vida, como educação, renda, água potável, saneamento e moradia adequada. Estudos em populações indígenas com longo contato com a sociedade envolvente na Amazônia Brasileira 10 e internacionalmente $5,7,11$ sugerem que a baixa estatura é mais prevalente nas áreas com maior privação de recursos sociais, indicadas por extrema pobreza 7 , falta de acesso à cidade por estradas 11, baixa escolaridade materna, analfabetismo acima dos 14 anos e porcentagem de crianças com baixo peso ao nascimento e desnutrição ${ }^{11}$. A baixa estatura também é mais prevalente nas famílias indígenas com menor renda 10 , mães com alta paridade (sete filhos ou mais) e em crianças com infecções respiratórias 11.0 inquérito nacional mexicano 7 , estudos chilenos 5 e de quatro países Andinos 6 evidenciaram 
maior prevalência de baixa estatura em crianças e de más condições de vida entre famílias com sobrenome indígena comparadas às de sobrenome espanhol, outro indicador de desigualdades sociais no estado nutricional no crescimento. Nenhum estudo encontrado examinou associação entre condições sociais e a estatura de crianças em população indígena, com longo contato com a sociedade envolvente, no Sul do Brasil.

O presente estudo descreve o estado nutricional dos menores de cinco anos, na sua primeira avaliação antropométrica no programa de controle da desnutrição e mortalidade das crianças na Terra Indígena Guarita (TI Guarita), no Rio Grande do Sul, Brasil, e examina a associação entre condições sociais e a estatura das crianças. Esse programa foi realizado de setembro de 2001 a novembro de 2002 pela Secretaria Estadual da Saúde do Rio Grande do Sul (SES-RS) e a organização não-governamental Conselho de Missão entre Índios (COMIN), com a participação das lideranças indígenas das 12 aldeias da TI Guarita e equipe de saúde que atuava na área 12 .

Esse programa originou-se no início de 2001, quando a SES-RS analisou a cobertura de serviços e a mortalidade na área do Distrito Sanitário Especial Indígena (DSEI) - Interior Sul situada no Rio Grande do Sul, comparando-as às médias estaduais 13. Essa análise evidenciou desigualdades étnicas em saúde: a taxa de consultas médicas e de hospitalização entre essa população era o dobro da média estadual e a mortalidade de menores de cinco anos era, no mínimo, cinco vezes maior que a média estadual. A mortalidade em menores de cinco anos entre os índios de etnia Kaingáng tem sido elevada 14, apresentando na TI Guarita taxas entre 97 e 168 óbitos para mil nascidos vivos, sendo a desnutrição associada a infecções relevante causa de morte 14,15 - entre 23 e $67 \%$ dos óbitos. Entretanto, apenas 25\% dessas mortes estavam notificadas no Sistema de Informação de Mortalidade 15, indicando que dados de saúde dessa população indígena estão sub-registrados no sistema oficial de informação em saúde.

Entre as 15 terras indígenas situadas no DSEI - Interior Sul, a TI Guarita, apresentava a pior situação de saúde em 2001. Parte da situação de saúde atual pode ser decorrente do processo de ocupação da terra e do processo civilizatório que vêm sofrendo. $\mathrm{O}$ aldeamento da TI Guarita foi criado em 1854, havendo desde então pressão da sociedade envolvente sob o modo tradicional de vida indígena e seu meio ambiente. A delimitação de quase $24 \mathrm{mil} \mathrm{hec-}$ tares do território atual em 1911 e sua demar- cação em 1918 ocorreu quando as demais terras da região, tradicionalmente ocupadas pelos índios para caça e coleta, foram liberadas para empresas de colonização. A partir da década de 40, empresas madeireiras desmataram a região e as terras posteriormente foram utilizadas para agricultura extensiva, prática que se estendeu à área demarcada 16. Atualmente a TI Guarita é habitada por índios de etnia Kaingáng (94,2\%) em 12 núcleos populacionais (aldeias), e Guaraní, todos com acesso por estrada. É limitada por uma rodovia estadual asfaltada e plantações mecanizadas de soja e trigo. A área está com a terra exaurida e contaminada por agrotóxicos, pouca mata nativa e fontes de água contaminadas por dejetos. Parte dos produtos tradicionalmente cultivados foi abandonada. A maioria das famílias sobrevive da agricultura familiar de subsistência, venda de artesanato, aposentadoria, trabalhos temporários e raros empregos de funcionário de órgão público 12,17. A renda familiar é baixa, em média inferior a dois salários mínimos 17,18. A realidade histórica de relações com a sociedade envolvente comprometeu as bases tradicionais de sustentabilidade, gerando dependência externa de produtos industrializados e de benesses de entidades, igrejas e do próprio estado, possivelmente afetando as condições de vida e saúde dessa população.

\section{Método}

O programa de controle da desnutrição e mortalidade implantado em 2001/2002, era por livre demanda e visava: (a) prover a cada criança o cartão de acompanhamento da saúde desenvolvido pelo Ministério da Saúde; (b) acompanhar trimestralmente o estado nutricional dos menores de cinco anos; (c) incluir as crianças desnutridas ou em risco de desnutrição e suas mães em uma intervenção nutricional; e (d) identificar fatores sociais associados à desnutrição das crianças, como subsídio para políticas e ações governamentais potencialmente efetivas para a melhoria da saúde dessa população indígena. A intervenção nutricional abrangia suplementação alimentar para a família baseada nos hábitos alimentares na TI Guarita, 12,18 e a realização de oficinas de nutrição com as mães e mulheres consideradas sábias pela comunidade. As oficinas buscavam construir práticas alimentares saudáveis considerando aspectos culturais e econômicos daquela população por meio da troca de conhecimento sobre saúde e alimentação saudável, preparação de alimentos e recuperação de receitas tra- 
dicionais. Condutas para favorecer a aceitação da comida pelas crianças, como reconhecer e o que fazer quando a criança não está bem de saúde também eram discutidas nessas oficinas.

Todos os menores de cinco anos da TI Guarita no período do programa eram elegíveis para este estudo. Os dados da primeira avaliação antropométrica da criança foram obtidos do banco de dados de acompanhamento das crianças e do inquérito realizado ao final do programa em fevereiro de 2003, no qual foram realizadas visitas domiciliares às famílias que não haviam sido incluídas. A medida do peso e da altura/comprimento das crianças (altura) foi realizada por nutricionistas e técnicas de enfermagem do programa, treinadas para incluir no programa aquelas com desnutrição ou risco nutricional - abaixo do percentil 10 do escore de peso para a idade da referencia do National Center for Health Statistics (NCHS) 19 - utilizando para isso o gráfico do Cartão da Criança e a metodologia, para a tomada das medidas, recomendada nas normas do Ministério da Saúde 20. O cálculo da idade da criança no dia da antropometria baseou-se na data de nascimento informada pela mãe e conferida no Cartão da Criança. Os índices de peso para idade (PIZ), altura para idade (AIZ) e peso para altura (PAZ) foram calculados em escores- $z$ do padrão americano CDC/2000 21 usando o módulo antropométrico do Epi Info 2002 (Centers for Disease Control and Prevention, Atlanta, Estados Unidos). Embora alguns autores questionem a adequação das curvas de referência internacionais, como as do NCHS, para populações indígenas alegando especificidades antropométricas dessas populações 22 , padrões internacionais têm sido recomendados pela Organização Mundial da Saúde para avaliação nutricional de crianças internacionalmente. A referência CDC/2000 foi utilizada neste estudo porque resultou de aprimoramento das técnicas estatísticas, ampliação da amostra e maior representatividade racial e diversidade étnica, comparado ao NCHS/ 197721.

As seguintes variáveis sociais foram obtidas do questionário aplicado à mãe biológica ou de criação, ao final do programa de intervenção nutricional: (a) provisão de recursos sociais indicados pela escolaridade materna, fonte de água para alimentação e destino dos dejetos; (b) condições econômicas e posse de utensílios domésticos para conservar alimentos indicados pela geladeira; (c) e variáveis maternas indicadas pela idade da mãe ao nascimento da criança mais velha entre os menores de cinco anos e pelo número de filhos menores de cinco anos. Essas variáveis têm sido amplamente uti- lizadas em estudos sobre desigualdade social e nutrição de crianças, principalmente em populações que vivem em situação de pobreza e exclusão social, em todo o mundo. A sua utilização neste estudo não pretende esgotar o tema da determinação social da nutrição em populações indígenas nem generalizar seu uso para outras etnias com diferentes processos históricos.

Das 1.296 crianças com menos de cinco anos durante o período do programa, $45 \%(n=583)$ tiveram somente uma avaliação antropométrica. Houve perda de informação de altura para $56(4,3 \%)$ crianças e do peso para 13 crianças (1\%) por falta da medida ou provável erro. As 1.296 crianças eram filhos de 708 mães. O número de filhos menores de cinco anos não teve perdas porque foi calculado com base na amostra. A falta de informação para as demais variáveis do questionário materno foi relativamente grande: escolaridade $(\mathrm{n}=136 ; 19,2 \%)$, água $(\mathrm{n}=$ $170 ; 24 \%)$, dejetos $(n=169 ; 23,9 \%)$, idade da mãe $(n=113 ; 16 \%)$ e geladeira $(n=150 ; 21,2 \%)$ e não ocorreu para todos os indivíduos nas mesmas variáveis. A maioria dessas perdas ocorreu porque o questionário foi aplicado ao final do programa e muitas famílias não foram encontradas, o que pode ser parcialmente explicado pelo período de sua aplicação, no verão, quando grande número de famílias se desloca para o litoral a fim de vender artesanato. As perdas variaram em torno de $20 \%$, mas provavelmente não alteraram fundamentalmente os resultados das associações entre as variáveis de exposição e a altura das crianças porque se distribuíram em todos os grupos de mães e porque a exclusão dos grupos sem informação em análises multivariadas foi evitada por meio da criação, em cada variável de exposição, de uma categoria específica para esses indivíduos.

A análise estatística foi realizada do seguinte modo: para descrever o estado nutricional das crianças, porcentagens de crianças com desnutrição foram apresentadas conforme idade e sexo. Escores menores de -2,0 desvios-padrão (dp) da mediana de referência foram definidos como indicadores de desnutrição nos índices PIZ, AIZ e PAZ, e escores maiores do que 2,00 no índice PAZ, como excesso de peso 1,21.

Para examinar os determinantes sociais da estatura, modelos de regressão linear foram usados para estimar a relação entre condições sociais e altura das crianças. Como a maioria das mulheres tinha mais de um filho menor de cinco anos e os filhos da mesma mãe tendem a ser mais parecidos entre si do que aqueles de mães diferentes, as observações, tanto das variáveis antropométricas como das variáveis de 
exposição, não são independentes. Uma vez que a técnica de regressão linear com o pacote estatístico SPSS (SPSS Inc., Chicago, Estados Unidos) pressupõe independência entre as observações, a opção de análise deste estudo foi realizar a regressão linear tendo como variável dependente a média dos escores AIZ dos filhos menores de cinco anos de cada mãe, e não o escore AIZ de cada criança. Inicialmente as médias de AIZ observadas em cada categoria de interesse foram apresentadas com a significância estatística (valores de p) para a diferença entre essas médias calculadas por análise de variância. A seguir, os modelos de regressão li- near brutos (não apresentados) e ajustados para confundimento foram estimados para cada categoria de exposição usando como referência a categoria de menor risco esperado, apresentado nos resultados. O coeficiente de regressão linear para cada categoria de interesse estima o quanto a média de AIZ das crianças difere da média da categoria de referência.

A escolha dos possíveis confundidores de cada variável (rodapé da Tabela 1) baseou-se no modelo hierárquico de determinação social da altura, o qual está esquematizado na Figura 1 , com as variáveis de exposição organizadas em três níveis hierárquicos. Nosso modelo pres-

Tabela 1

Média e intervalo de confiança de 95\% (IC95\%) do escore de AIZ * para idade dos filhos menores de cinco anos e coeficientes de regressão linear das diferenças entre as médias para as variáveis estudadas.

\begin{tabular}{|c|c|c|c|c|}
\hline & \multirow[t]{2}{*}{ Variável de exposição } & \multirow[t]{2}{*}{ Mães (n) } & \multicolumn{2}{|c|}{ AIZ } \\
\hline & & & Média & 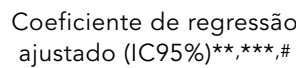 \\
\hline \multirow[t]{12}{*}{ 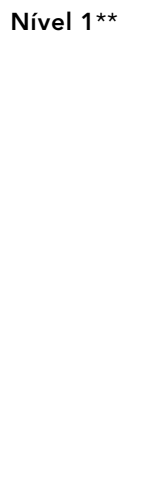 } & Escolaridade materna (em anos) $)^{\star \star}$ & & & \\
\hline & $<1$ & 196 & $-1,60$ & $-0,17(-0,35-0,02)$ \\
\hline & 1 e mais & 376 & $-1,39$ & Referência \\
\hline & & & $p=0,023$ & $p=0,083$ \\
\hline & Origem da água para alimentação** & & & \\
\hline & Fonte, poço ou rio & 358 & $-1,58$ & $-0,20(-0,01--0,40)$ \\
\hline & Torneira & 180 & $-1,34$ & Referência \\
\hline & & & $p=0,011$ & $p=0,046$ \\
\hline & Destino dos dejetos ${ }^{\star *}$ & & & \\
\hline & Terreiro, mato, latrina & 483 & $-1,54$ & $-0,20(-0,10-0,50)$ \\
\hline & Sanitário com descarga & 56 & $-1,21$ & Referência \\
\hline & & & $p=0,028$ & $p=0,195$ \\
\hline \multirow[t]{8}{*}{ Nível $2^{\star \star \star}$} & Geladeira*** & & & \\
\hline & Não & 466 & $-1,55$ & $-0,33(-0,62--0,05)$ \\
\hline & Sim & 73 & $-1,11$ & Referência \\
\hline & & & $p=0,001$ & $p=0,021$ \\
\hline & Idade materna (em anos) ${ }^{\star \star \star}$ & & & \\
\hline & $<16$ & 62 & $-1,60$ & $-0,35(-0,64--0,06)$ \\
\hline & $\geq 16$ & 533 & $-1,45$ & Referência \\
\hline & & & $p=0,027$ & $p=0,019$ \\
\hline \multirow[t]{5}{*}{ Nível 3\# } & Número de filhos menores de cinco anos\# & & & \\
\hline & 1 & 311 & $-1,35$ & Referência \\
\hline & 2 & 235 & $-1,49$ & $-0,08(-0,29-0,13)$ \\
\hline & 3 e mais & 162 & $-1,46$ & $0,01(-0,22-0,25)$ \\
\hline & & & $p=0,334$ & $p=0,826$ \\
\hline \multicolumn{2}{|c|}{ Total da amostra } & 708 & $-1,42$ & \\
\hline
\end{tabular}

AIZ = índice de altura para idade

* Em escores $Z$ da referência americana CDC/2000.

** Nível 1: modelo inclui escolaridade materna, origem da água para alimentação e despejo dos dejetos.

*** Nível 2: ajustado para escolaridade materna, origem da água para alimentação e demais variáveis deste nível.

\# Nível 3: ajustado para escolaridade materna, origem da água para alimentação, geladeira e idade materna.

\#\# Idade materna ao nascimento do filho mais velho entre os menores de cinco anos.

Nota: Para as demais variáveis, exceto número de filhos, o total da amostra é menor do que 708 devido

à perda de informações. 
Figura 1

Modelo hipotético dos determinantes sociais da altura na criança.

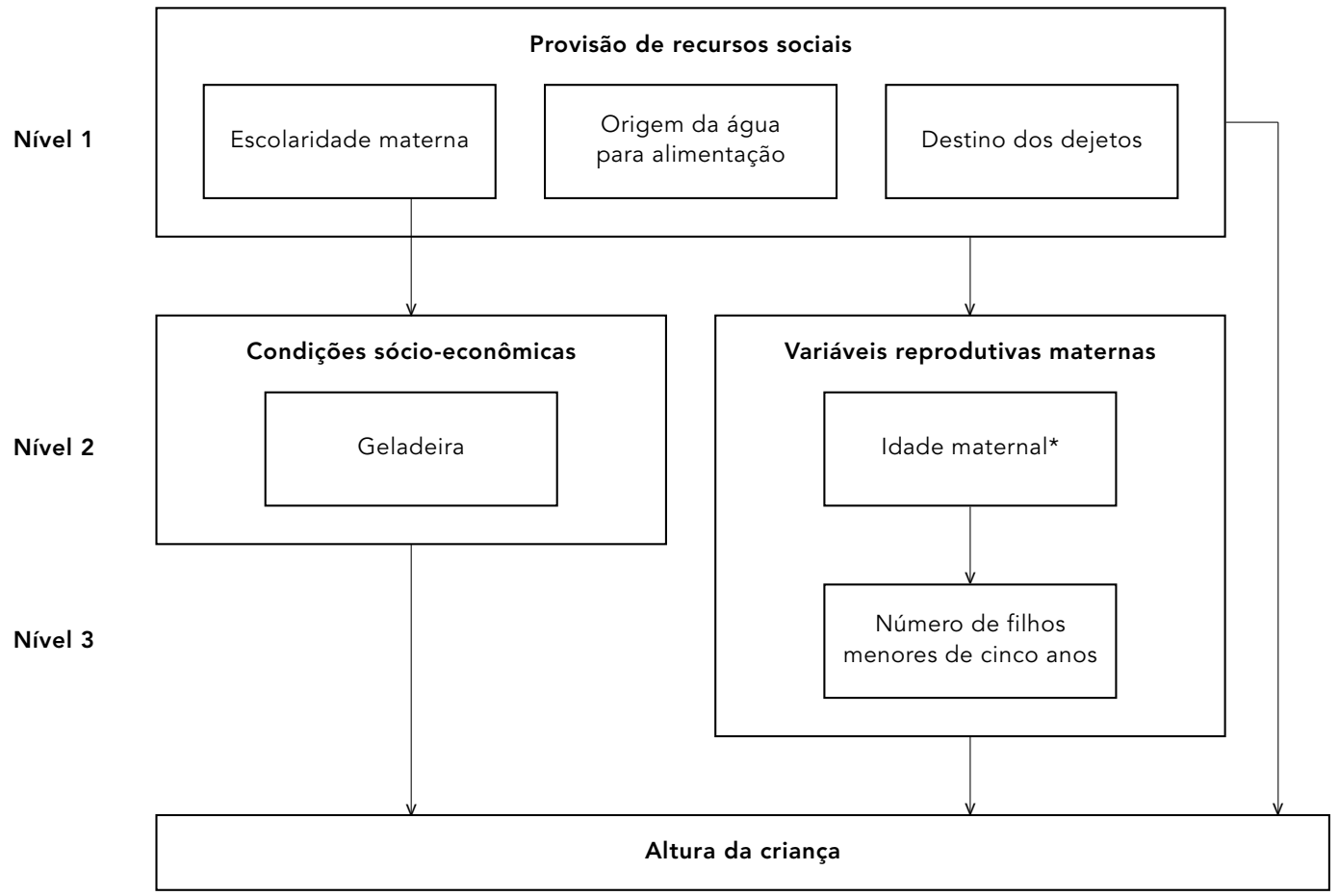

* Ao nascimento da criança mais velha entre os menores de cinco anos.

supõe que a determinação social da nutrição entre este grupo indígena, que vive em contato antigo e intenso com a sociedade envolvente, pode ser, pelo menos em parte, explicada pelo modelo de determinação social do processo de saúde amplamente utilizado em estudos na área da saúde em diversos grupos sociais e etnias internacionalmente. Este modelo prediz que a privação de recursos sociais - escolaridade, água encanada e sanitário com descarga (nível 1), propicia doenças infecciosas e parasitárias recorrentes que debilitam a saúde da criança e afetam seu crescimento. Aqui a escolaridade não está em um nível diferente da provisão de água e saneamento nem depende da renda, como na maioria dos demais estudos, porque a escolaridade e os demais recursos sociais para as populações indígenas aldeadas são providos unicamente pelo estado. As escolas iniciaram sua atuação dentro da área na década de 60 oferecendo primeiro grau bilíngüe. Parte do efeito negativo da privação de recursos sociais sobre o crescimento decorre da maior probabilidade das mulheres analfabetas não terem geladeira para conservar alimentos, de serem mães antes dos 16 anos de idade (nível 2) e apresentarem intervalo interpartal curto, levando a maior número de filhos menores de cinco anos (nível 3). A baixa idade materna afeta o crescimento da criança possivelmente, em parte, porque essas mães tendem a ter menor habilidade e menor comprometimento com o cuidado da criança, mesmo levando em conta os recursos sociais disponíveis e as piores condições sócio-econômicas indicadas pela posse de geladeira, como nas análises multivariadas de nível 2 deste estudo. Contudo, a habilidade e comprometimento com o cuidado da criança não foram avaliados neste estudo. Nos modelos multivariados foram incluídos como possíveis confundidores as demais variáveis do mesmo nível hierárquico e aquelas de nível mais alto, que evidenciaram associação com a altura no seu respectivo modelo multivariado $(p<0,20)$. Foi preestabelecido o nível de $5 \%(\mathrm{p}<0,05)$ para considerar estatisticamente significante o efeito de uma exposição sobre a altura e o nível de $10 \%(p<0,10)$ para significância limítrofe. 
Como nos modelos multivariados, a falta de informação de uma variável leva a exclusão do indivíduo e havia perda de informação na maioria das variáveis; uma categoria "sem informação" foi criada para cada variável. A variável contendo a categoria "sem informação" foi incluída nos modelos de regressão em que a variável de origem era possível confundidor de alguma das demais.

Este estudo é parte da avaliação da intervenção realizada pela SES-RS e COMIN, solicitada a uma das autoras (M.L.D.) com garantia ética e criminal de sigilo dos nomes que constam no banco de dados e direito de publicação. Os resultados do estudo foram entregues às instituições envolvidas e apresentados aos gestores da saúde indígena, comunidade indígena e equipe de saúde.

\section{Resultados}

A distribuição das crianças por idade e sexo (Tabela 2) mostra discreto predomínio de meninas e maior proporção de menores de um ano (note que o estudo incluiu todas as crianças que nasceram no período do programa). A distribuição das variáveis do questionário materno sugere que $34,3 \%$ (196/572) das mulheres nunca freqüentaram escola ou tinham menos de um ano de escolaridade; $40,2 \%(230 / 572)$ tinham de 1 a 4 anos de escolaridade e apenas $5,2 \%(30 / 572)$ completaram o ensino fundamental. As condições de vida da maioria das famílias dessa população indígena eram precárias: $66,5 \%$ (358/538) das mães referiram que a família consumia água diretamente de fonte, poço ou rio; $89,6 \%$ (483/539) depositavam dejetos no terreiro, mato ou latrina; $86,9 \%$ (485/ 558) não tinham geladeira para conservar alimentos; 10,4\% (62/595) tinham menos de 16 anos ao nascimento da criança mais velha entre os filhos menores de cinco anos; $27,9 \%$ (166/ 595) de 16 a 19 anos; $43,9 \%$ (311/708) tinham um filho; $33,2 \%$ (235/708) dois filhos; e 22,9\% $(162 / 708)$ três ou mais filhos menores de cinco anos (resultado não apresentado em tabela).

\section{O estado nutricional das crianças}

Cerca de $34,7 \%$ das crianças apresentaram baixa estatura; $12,9 \%$ baixo PIZ e $4,2 \%$ baixo PAZ (Tabela 1$) ; 8,7 \%(\mathrm{n}=107)$ apresentaram excesso de PAZ e $1 \%(n=13)$ excesso de PIZ (resultado não apresentado em tabela). A baixa estatura foi mais prevalente entre os meninos $\left(\chi^{2} ; \mathrm{p}=\right.$ $0,001)$ e após o primeiro ano de idade $\left(\chi^{2} ; \mathrm{p}<\right.$ $0,001)$, e o baixo PIZ, em crianças de um e dois $\operatorname{anos}\left(\chi^{2} ; \mathrm{p}<0,001\right)$. Não houve evidência de diferença entre os sexos e faixa etária na prevalência de baixo PAZ (Tabela 2). A Figura 2 mostra que a altura das crianças da TI Guarita tende a ser menor do que a das crianças de mesma idade e sexo na população de referência. Dentre as crianças que tiveram somente uma avaliação durante o programa, $17,5 \%$ apresentavam o critério de inclusão no programa, ou seja, estavam abaixo do percentil 10 no índice de PIZ (8,3\% risco nutricional $-\mathrm{n}=101$; e 9,2\% $\mathrm{n}=52$ desnutrição), 35\% $(\mathrm{n}=201)$ apresentavam baixa estatura e 2,9 $(\mathrm{n}=16)$ baixo PAZ. Cerca de metade das mães $(48,5 \%)$ tinha um ou mais filhos desnutridos.

\section{Determinantes da altura das crianças}

As médias de AIZ foram menores quando a mãe nunca freqüentou a escola ou estudou menos de um ano ( $\mathrm{p}=0,023)$, a água para alimentação era coletada em fonte, poço ou rio $(p=0,011)$, os dejetos eram depositados no mato, terreiro ou latrina $(0,028)$ e não havia geladeira $(p=0,001)$. Não houve evidência de diferença entre as médias de AIZ para a variável idade materna ao nascimento do mais velho entre os filhos menores de cinco anos $(p=0,277)$ e número de filhos menores de cinco anos ( $\mathrm{p}=$ 0,334 ) (Tabela 1).

O modelo de regressão linear correspondente ao primeiro nível da Figura 1 - "escolaridade materna", "origem da água para alimentação" e "destino dos dejetos" - sugere que as crianças tendem a ser menores quando a "origem da água para alimentação” é coletada em fonte, poço ou rio do que quando é utilizada a água da torneira rotineiramente, mesmo levando em conta a escolaridade materna e o destino dos dejetos $(\mathrm{p}=0,046)$ (Tabela 1$)$. O coeficiente de regressão para "escolaridade mater$n a$ " menor do que um ano sugere que a altura tende a ser menor entre os filhos dessas mulheres comparados às demais, embora a significância estatística tenha sido limítrofe $(p=0,083)$, após ajuste para "origem da água para alimentação" e "destino dos dejetos" (Tabela 1). O coeficiente de regressão linear bruto para a variável "destino dos dejetos" mostrou que a altura das crianças era, em média, menor quando os dejetos da família eram eliminados no ambiente imediato (terreiro, mato, latrina) (Tabela 1). O efeito negativo da eliminação dos dejetos no ambiente imediato diminuiu e perdeu a significância estatística $(p=0,195)$ quando a escolaridade materna e a origem da água utilizada para alimentação foram levadas em conta (Tabela 1). 
Distribuição e porcentagem de crianças com desnutrição na primeira avaliação feita pelo programa de controle da desnutrição e mortalidade de crianças da Terra Indígena Guarita, conforme sexo e faixa etária. Rio Grande do Sul, Brasil, 2001/2002.

\begin{tabular}{|c|c|c|c|c|c|c|}
\hline & \multicolumn{2}{|c|}{$\mathrm{PIZ}$} & \multicolumn{2}{|c|}{ AIZ } & \multicolumn{2}{|c|}{ PAZ } \\
\hline & $\begin{array}{c}\text { Crianças } \\
\text { avaliadas (n) }\end{array}$ & 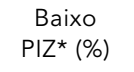 & $\begin{array}{c}\text { Crianças } \\
\text { avaliadas (n) }\end{array}$ & $\begin{array}{c}\text { Baixa } \\
\operatorname{AlZ}^{\star \star}(\%)\end{array}$ & $\begin{array}{c}\text { Crianças } \\
\text { avaliadas (n) }\end{array}$ & $\begin{array}{c}\text { Baixo } \\
\left.\text { PAZ*** }^{*} \%\right)\end{array}$ \\
\hline \multicolumn{7}{|c|}{ Idade (em anos) } \\
\hline$<1$ & 382 & 10,5 & 369 & 22,5 & 368 & 3,5 \\
\hline 1 e 2 & 486 & 18,9 & 470 & 42,3 & 457 & 5,0 \\
\hline \multirow[t]{2}{*}{$3 e+$} & 415 & 8,2 & 401 & 37,3 & 400 & 4,0 \\
\hline & & $p<0,001$ & & $p<0,001$ & & $p=0,556$ \\
\hline \multicolumn{7}{|l|}{ Sexo } \\
\hline masculino & 619 & 14,2 & 597 & 39,4 & 592 & 4,1 \\
\hline \multirow[t]{2}{*}{ feminino } & 664 & 11,7 & 643 & 30,3 & 633 & 4,4 \\
\hline & & $p<0,109$ & & $p=0,001$ & & $p<0,430$ \\
\hline Total & 1.283 & $166(12,9)$ & 1.240 & $430(34,7)$ & 1.225 & $52(4,2)$ \\
\hline
\end{tabular}

$\mathrm{PIZ}=$ índice de peso para idade; $\mathrm{AIZ}=$ índice de altura para idade; $\mathrm{PAZ}=$ peso para altura.

* $\mathrm{PIZ}<-2,0$ escores Z da referência CDC/2000.

** $\mathrm{AIZ}<-2,0$ escores $Z$ da referência CDC/2000.

*** PAZ $<-2,0$ escores $Z$ da referência CDC/2000.

Figura 2

Distribuição dos escores de altura para idade (AIZ) das crianças.

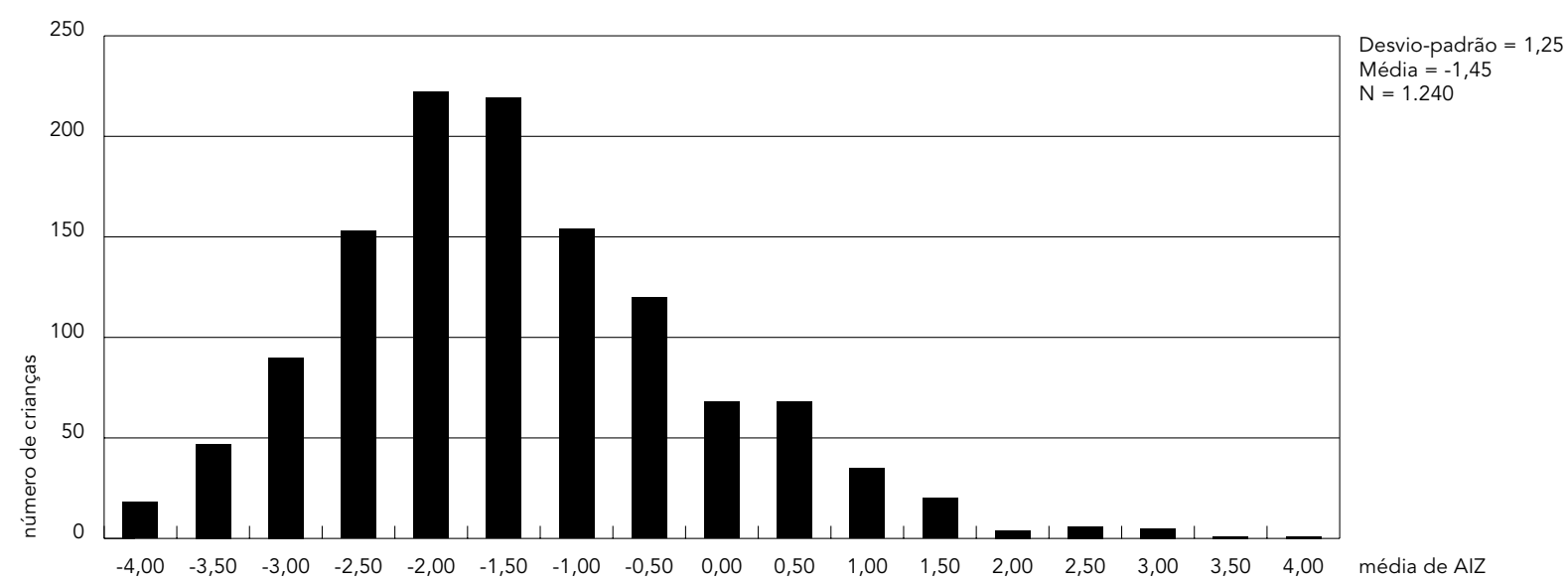


O modelo de regressão linear correspondente ao segundo nível da Figura 1 - "idade mater$n a$ " (ao nascimento da criança mais velha entre os menores de cinco anos e posse de geladeira) - sugere que a altura da criança é, em média, menor a quando a mãe tem menos de 16 anos ( $\mathrm{p}=0,019)$ e não há geladeira para conservação dos alimentos ( $\mathrm{p}=0,028)$. Esse modelo multivariado incluiu "escolaridade materna", “origem da água para alimentação", "destino dos dejetos" e as duas variáveis desse nível (Tabela 1 ).

O modelo de regressão para o terceiro nível da Figura 1 - "número de filhos menores de cinco anos" - não evidenciou efeito dessa variável sobre a altura média dos filhos, tanto no modelo de regressão bruto (Tabela 1) como no modelo ajustado para confundimento da escolaridade materna, origem da água utilizada para alimentação, idade materna ao nascimento da criança mais velha entre os menores de cinco anos e posse de geladeira $(\mathrm{p}=0,826)$ (Tabela 1$)$.

\section{Discussão}

Este estudo teve como objetivos descrever o estado nutricional das crianças menores de cinco anos da TI Guarita em 2001/2002 e estudar determinantes sociais do crescimento linear, indicador das condições de vida e saúde da população. A determinação da saúde desse povo indígena é inseparável do processo de colonização do Sul do Brasil e suas conseqüências sobre a ocupação da terra, a deterioração do meio ambiente (desmatamento, esgotamento da terra e das fontes de água, contaminação por agrotóxicos) e a modificação do modo tradicional de vida indígena (sedentarização, cristianização, dependência de bens manufaturados roupas, ferramentas, utensílios - e alimentos industrializados).

Nesse contexto, a altura das crianças pode ser um indicador, não apenas do estado nutricional, mas também das condições de vida e saúde na TI Guarita. Tendo as mães, biológica ou social, como foco da atenção do programa de controle da desnutrição e mortalidade das crianças, a avaliação buscou investigar as condições de vida associadas à menor altura dos filhos utilizando dados secundários. Embora o uso de dados secundários imponha limitações ao desenho e à análise estatística, é de baixo custo e tem grande relevância social porque gera conhecimento com informação produzida pelos e para os serviços. As variáveis estudadas representam dimensões importantes da qualidade de vida das famílias e têm se mostra- do fortemente associadas à saúde da criança e seu estado nutricional em outros estudos. A porcentagem de perda de informação em cada uma das variáveis de exposição de interesse foi relativamente grande (até $24 \%$ ), o que indica a necessidade de qualificar as informações produzidas pelos serviços.

\section{Estado nutricional de crianças indígenas}

Na revisão realizada não foram encontrados estudos sobre o padrão de crescimento utilizando a referência CDC/2000, mas os estudos que utilizaram a referência NCHS/1977 19 para comparação também encontraram taxas muito altas de desnutrição em crianças. Os resultados deste estudo mostram que, em 2001/2002, cerca de um terço das crianças $(34,7 \%)$ apresentava baixa AIZ, $12,9 \%$ baixo PIZ e $4,2 \%$ baixo PAZ. Altos níveis de desnutrição já foram apontados em 1995, quando um inquérito nutricional de menores de cinco anos nessa mesma Terra Indígena mostrou $47,4 \%$ de baixa AIZ, $15,6 \%$ baixo PIZ e 2,1\% baixo PAZ 17. Estudos recentes apontam a desnutrição de crianças indígenas como grave problema de saúde pública, onde 16 a 53,5\% das crianças apresentavam baixa estatura 10,23,24,25,26. As prevalências de desnutrição nas crianças indígenas brasileiras, relatadas neste e em outros estudos, parecem ser bem maiores do que as observadas em estudos realizados na população em geral como, por exemplo, no Rio Grande do Sul, onde estudos de base populacional encontraram prevalência de baixa estatura de $6,8 \%{ }^{8}$. Os poucos estudos que comparam as condições de saúde das populações indígenas aos demais segmentos da sociedade no Brasil 27 e internacionalmente, 5,6,7 também mostram que as crianças indígenas apresentam piores condições de saúde do que as demais. A manutenção de altas taxas de desnutrição nas populações indígenas enquanto há diminuição da prevalência de desnutrição no Brasil 3 e internacionalmente 28,29, sugerem que as desigualdades em saúde são predominantemente sociais.

As crianças maiores de um ano apresentaram maior prevalência de desnutrição crônica, com maior taxa de desnutrição aguda entre um e dois anos. Apesar da inexistência de dados sobre aleitamento materno e dieta nessa faixa etária entre Kaingang, é possível que as menores taxas de desnutrição entre os menores de um ano sejam decorrentes da manutenção desse hábito e, em parte, ao menor contato com o meio ambiente imediato. No momento em que começa a caminhar e explorar o ambiente imediato a criança fica mais exposta a contrair pa- 
rasitoses, desenvolver infecções e receber alimento insuficiente ou deficiente na dieta. Por outro lado, a prevalência de quase $10 \%$ de excesso de peso para altura em crianças menores de cinco anos é preocupante. O índice de peso para estatura foi cinco vezes maior neste estudo quando comparado à avaliação realizada em 1996. Em uma população com alta prevalência de baixa estatura, dietas inadequadas somadas a mecanismos fisiológicos compensatórios encontrados em populações sujeitas à desnutrição poderão aumentar a prevalência de obesidade e suas conseqüências em relação ao maior risco de doenças crônico-degenerativas.

As condições de vida associadas à menor altura dos filhos

Como esperado, o analfabetismo materno $(<1$ ano de escolaridade) e a maternidade em idade muito precoce (menor de 16 anos), tiveram efeito negativo sobre a altura das crianças da TI Guarita, embora a significância estatística da escolaridade tenha sido limítrofe. Não foram encontrados estudos de determinantes de altura considerando escolaridade e idade materna em populações indígenas. Estudos na população em geral mostram que a baixa escolaridade materna e a gravidez na adolescência - mais freqüentes em famílias em privação sócio-econômica - aumentam o risco de retardo de crescimento intra-uterino e de prematuridade ao nascimento, fatores de risco para adoecimento nos primeiros anos de vida, déficit de altura e morte antes dos cinco anos de idade $28,29,30$. O espaço escolar pode ter possibilitado maior acesso à informação e interpretação do processo saúde/doença após a destituição dos kuiã (xamã) 16, demonizados como feiticeiros pelas igrejas. É possível que a escolaridade da mãe, nesse grupo indígena, seja um indicador de que a capacidade de ler e escrever resulta em vantagem nutricional para a família, que necessita ser investigado.

Outros estudos na população em geral sugerem que a baixa idade materna coloca a criança em maior risco de morbidade e déficits de crescimento, principalmente devido às privações sócio-econômicas a que essas mulheres tendem a estar submetidas 30,31, mas também pela menor disponibilidade materna para o cuidado da criança 32 . Tradicionalmente nas populações indígenas as mulheres têm filhos antes dos vinte anos. Contudo, 16 anos parece ser uma idade muito precoce entre esses índios pois ocorreu para $10 \%$ dessas mulheres e associou-se à menor altura da criança. Em 1996, estudo realizado entre a população indígena do
Estado refere que $80 \%$ das mulheres pesquisadas tiveram o primeiro filho antes dos vinte anos de idade 17 . Neste estudo, $38,3 \%$ das mães tinham menos de vinte anos ao nascimento da criança mais velha entre os menores de cinco anos, sugerindo que as mulheres têm postergado a primeira gestação nos últimos dez anos. É possível que ocorra reestruturação na composição das famílias, de extensas para nucleares, diminuindo os cuidadores entre os casais jovens, porém tais hipóteses necessitam ser investigadas em outros estudos.

Neste estudo não houve evidência de efeito do número de filhos menores de cinco anos sobre a altura das crianças. Esse resultado difere do encontrado em estudos realizados na população em geral, os quais apontam que a altura tende a ser menor e o risco de baixa estatura maior nas famílias com maior número de filhos ou crianças menores de cinco anos 33 . Estudo em população indígena também encontrou associação entre baixa estatura e maior número de filhos 11. É possível que o número de crianças não predisponha à menor altura na TI Guarita porque há muitas famílias extensas, com mais de uma geração vivendo em um domicílio, muitas vezes também com sobrinhos e tios 17,18, o que poderia aumentar o número de cuidadores das crianças. Na ausência dos pais, avós (kofá) e tias costumam assumir a responsabilidade pelas crianças, neste estudo, consideradas mães sociais.

Estudos têm mostrado que moradia inadequada com falta de água potável e de saneamento básico é um dos maiores determinantes de retardo de crescimento internacionalmente, pois aumentam o risco de infecções, principalmente respiratórias e diarréia 33,34 , e de apresentá-las em maior gravidade, indicada pela hospitalização 9,34 e morte. No Rio Grande do Sul, na TI Guarita e outras áreas indígenas do estado 13,14, essas doenças têm sido as principais causas de hospitalizações e morte em menores de cinco anos. Em populações indígenas, precárias condições de saneamento e água contaminada propiciam o poliparasitismo $35 \mathrm{e}$ infecções recorrentes que, associadas a situações de carência alimentar, podem levar à desnutrição. Os resultados deste estudo sugerem que essa população vive em condições de saneamento precárias e o preparo de alimentos com água de poço, fonte ou rio, quando comparados aos que utilizam água de torneira, colocam a criança em risco de desnutrição afetando seu crescimento linear. No entanto, os dejetos depositados diretamente no meio ambiente imediato não evidenciaram efeito quando o modelo de regressão levou em conta a es- 
colaridade materna e a origem da água utilizada no preparo dos alimentos. É possível que a falta de evidência de efeito do destino dos dejetos deva-se à contaminação do ambiente em geral pelos dejetos depositados pelas demais famílias $(90 \%)$ no ambiente, o que afeta também as famílias com saneamento básico, não evidenciando uma possível associação com baixa estatura. A falta de geladeira - indicador sócio-econômico - coloca a criança em risco de menor crescimento linear devido, em parte, ao efeito sobre a conservação dos alimentos, e provavelmente porque também indica maior acesso a outros bens materiais que favorecem a qualidade de vida.

Estudos utilizando dados primários e com delineamento transversal, de coorte e ensaios randomizados controlados que investiguem detalhadamente os diferentes níveis de determinação do processo do crescimento e nutrição nessa população são necessários para confirmar e melhor entender os resultados aqui apresentados. Contudo, os resultados deste estudo sugerem que é necessário implantar políticas para acompanhamento e promoção do crescimento e vigilância nutricional - além da desnutrição, o excesso de peso das crianças emerge como problema de saúde podendo ter grandes implicações sobre a saúde no futuro. Intervenções na nutrição devem ser pensadas com a comunidade, especialmente com as mulheres indígenas, e devem acompanhar-se de estudos com dados primários delineados para avaliação de sua efetividade, uma vez que a efetividade de intervenções nutricionais é pouco conhecida em populações indígenas.

Provisão de educação, água potável encanada e sanitários são reivindicações da população da TI Guarita e este estudo mostra a importância que a provisão de recursos sociais públicos podem ter ao romper o ciclo de contaminação ambiental, diminuindo o risco de infecções recorrentes e poliparasitismo. Embora não tenha coletado dados sobre o consumo alimentar e a qualidade dos serviços de saúde, os quais são de extrema relevância na determinação da saúde e do estado nutricional de populações vulneráveis, há evidência de que estes são insatisfatórios na TI Guarita 12,18. Priorizar, na assistência à saúde, ações potencialmente efetivas para promover a saúde infantil - como prevenção, detecção e tratamento precoce de parasitoses e infecções - oferecendo atenção especial às adolescentes, ao pré-natal, parto e acompanhamento da saúde da criança, poderá atenuar os índices de desnutrição e mortalidade encontrados.

A busca de soluções para as precárias condições de vida a que estão sujeitas algumas populações indígenas vincula-se a fenômenos de exclusão social complexos, e diminuir a iniqüidade em saúde a que estão submetidos 36 envolve abordagens onde a discriminação seja trazida à luz superando a exclusão histórica e seu impacto sobre a saúde dessas populações.

\section{Resumo}

O estado nutricional de crianças menores de cinco anos e fatores associados à estatura foram estudados usando dados de um programa para controle da desnutrição e mortalidade na Terra Indígena Guarita, Rio Grande do Sul, Brasil, 2001/2002. Índices antropométricos foram calculados em escores- $z$ da referência CDC/2000. Na primeira avaliação pelo programa, $34,7 \%$, das crianças apresentavam baixa estatura, $12,9 \%$ baixo peso para idade, 4,2\% baixo peso para estatura e 8,7\% sobrepeso. Baixa estatura foi mais prevalente em meninos e maiores de um ano. Modelos de regressão linear múltipla sugerem que a altura foi, em média, menor quando a água para alimentação era de

fonte/poço/rio ( $p=0,046)$, não havia geladeira para conservar alimentos ( $p=0,021)$, a mãe era menor de 16 anos ao nascimento do mais velho entre os filhos menores de cinco anos ( $p=0,019)$ e analfabeta $(p=$ $0,083)$. O destino dos dejetos evidenciou efeito apenas no modelo bruto. Não houve evidência de efeito do número de filhos menores de cinco anos. Políticas de inclusão social e provisão de recursos sociais e de saúde são potencialmente relevantes para a saúde e nutrição nessa população.

Estado Nutricional; Iniqüidade Social; Índios Sulamericanos; Criança 


\section{Colaboradores}

I. A. Menegolla e M. L. Drachler participaram da elaboração do questionário de encerramento do programa da intervenção juntamente com os demais autores, da análise de consistência dos dados e da redação do artigo. I. H. Rodrigues e L. R. Schwingel elaboraram e implementaram o projeto de intervenção nutricional, coordenaram a coleta dos dados e participaram da revisão crítica do artigo. M. B. Pedroso e E. Scapinello contribuíram na elaboração do questionário de encerramento do programa da intervenção juntamente com os demais autores, treinamento da equipe de campo, coordenação da digitação dos dados e colaboraram no controle de qualidade dos dados. J. C. C. Leite colaborou na elaboração do questionário de encerramento do programa da intervenção, na análise dos dados e na redação do artigo.

\section{Agradecimentos}

Às mães e lideranças da Terra Indígena Guarita, pelo seu esforço em superar a herança de adversidades, exclusão e discriminação e criar um futuro para seus filhos; à Equipe de Campo do Conselho de Missão entre Índios (COMIN) que realizou a intervenção e a coleta de dados: Eloí Alberto, Sandra Claudino, Ilani Dettenborn, Noili Falcade, Ingret Kaminski, Verlaine Petri, Laísa Sales Ribeiro; e à Equipe do Programa de Alimentação e Nutrição/Secretaria Estadual da Saúde do Rio Grande do Sul (SES-RS) que exaustivamente revisou e digitou os dados: Rafael Bender, Maria Alice Kantmann e Deise Valírio Vetcomilla.

\section{Referências}

1. World Health Organization. Physical status: the use and interpretation of anthropometry. Geneva: World Health Organization; 1995 (Technical Report Series, 854).

2. Preberg Z. Changes in growth patterns in Zagreb school children related to socioeconomic background over the period 1973-1991. Ann Hum Biol 1998; 25:425-39.

3. Kac G. Tendência secular em estatura: uma revisão de literatura. Cad Saúde Publica 1999; 15:45161.

4. Eisenmann JC, Katzmarzyk PT, Arnall DA, Kanuho V, Interpreter C, Malina RM. Growth and overweight of Navajo youth: secular changes from 1955 to 1997. Int J Obes Relat Metab Disord 2000; 24:211-8.

5. Amigo H, Erazo M, Bustos P. Altura de padres e hijos chilenos de diferente etnia y vulnerabilidad social. Salud Pública Méx 2000; 42:504-10.

6. Larrea C, Freire W. Social inequality and child malnutrition in four Andean countries. Rev Panam Salud Pública 2002; 11:356-64.

7. Rivera JA, Monterrubio EA, González-Cossío T, García-Ferregrino R, García-Guerra A, SepúlvedaAmor J. Nutritional status of indigenous children younger than five years age in México: results of a national probabilistic survey. Salud Pública Méx 2003; 45(4 Suppl):S466-76.

8. Aerts D, Drachler ML, Giugliani ERJ. Determinants of growth retardation in Southern Brazil. Cad Saúde Pública 2004; 20:1182-90.
9. Waterlow JC. Introduction. Causes and mechanisms of linear growth retardation (stunting). Eur J Clin Nutr 1994; 48:S1-4.

10. Ribas DLB, Sganzerla A, Zorzatto JR, Philippi ST. Nutrição e saúde infantil em uma comunidade indígena Teréna, Mato Grosso do Sul, Brasil. Cad Saúde Pública 2001; 17:323-31.

11. Buitrón D, Hurtig AK, Sebastián MS. Estado nutricional em niños napurunas menores de cinco anos en la Amazonia ecuatoriana. Rev Panam Salud Pública 2004; 15:151-9.

12. Conselho de Missão entre Índios, Secretaria da Saúde do Estado do Rio Grande do Sul. Projeto de nutrição Terra Indígena Guarita. Porto Alegre: Conselho de Missão entre Índios, Secretaria da Saúde do Estado do Rio Grande do Sul; 2001.

13. Diehl EE, Drachler ML, Menegolla IA, Rodrigues IH. Financiamento e atenção à saúde no Distrito Sanitário Especial Indígena Interior Sul. In: Coimbra Jr. CEA, Santos RV, Escobar AL, organizadores. Epidemiologia e saúde dos povos indígenas no Brasil. Rio de Janeiro: Editora Fiocruz; 2003. p. 217-34.

14. Hökerberg IHM. A situação de saúde dos índios Kaingang do Rio Grande do Sul, 1985-1995 [Dissertação de Mestrado]. Rio de Janeiro: Escola Nacional de Saúde Pública, Fundação Oswaldo Cruz; 1997.

15. Menegolla IA, Fukuoka E, Rodrigues IH. Investigação de óbitos de crianças menores de 5 anos da Terra Indígena Guarita, Município de Redentora/RS. Rev Bras Epidemiol 2002; 3 Suppl:229. 
16. Marcon T. Dados atuais sobre as reservas no Rio Grande do Sul. In: Marcon T, organizador. História e cultura Kaingang no Sul do Brasil. Passo Fundo: Editora da Universidade de Passo Fundo; 1994.

17. Coordenação Regional do Rio Grande do Sul, Fundação Nacional de Saúde, Ministério da Saúde. Diagnóstico de saúde da população indígena do Rio Grande do Sul. Porto Alegre: Coordenação Regional do Rio Grande do Sul, Fundação Nacional de Saúde, Ministério da Saúde; 1996.

18. Schuch I. Perfil socioeconômico e alimentar das famílias indígenas Kaingang de Guarita, RS [Dissertação de Mestrado]. Campinas: Universidade de Campinas; 2001.

19. National Center for Health Statistics. Growth curves for children, birth-18 years. Department of Health, Education and Welfare. Hyattsville: National Center for Health Statistics; 1977. (Series 11, 165).

20. Ministério da Saúde. Acompanhamento do crescimento e desenvolvimento infantil. Brasília: Ministério da Saúde; 2001.

21. Kuczmarski RJ, Ogden CL, Grummer-Strawn LM, Flegal KM, Guo SS, Wei R, et al. CDC growth charts: United States. Adv Data 2000; 8:1-27.

22. Holmes R. Small is adaptative: nutritional anthropometry of native Amazonians. In: Sponsel LE, editor. Indigenous peoples and the future of Amazonia: an ecological anthropology of an endangered world. Tucson: University of Arizona Press; 1995. p. 121-48.

23. Escobar AL, Santos RV, Coimbra Jr. CEA. Avaliação nutricional de crianças indígenas Pakaánova (Wari'), Rondônia, Brasil. Rev Bras Saúde Matern Infant 2003; 3:457-61.

24. Martins SJ, Menezes RC. Evolução do estado nutricional de menores de cinco anos em aldeias indígenas da tribo Parakanã, na Amazônia Oriental Brasileira (1989-1991). Rev Saúde Pública 1994; 28:1-8.

25. Capelli JCS, Koifman S. Avaliação do estado nutricional da comunidade indígena Parkatêjê, Bom Jesus do Tocantins, Pará, Brasil. Cad Saúde Pública $2001 ; 17: 433-7$.

26. Gugelmin AS. Nutrição e alocação de tempo dos Xavantes de Pimentel Barbosa, Mato Grosso: um estudo em ecologia humana e mudanças [Dissertação de Mestrado]. Rio de Janeiro: Escola Nacional de Saúde Pública, Fundação Oswaldo Cruz; 1995.
27. Vieira AA, Braga JU, Moraes CL. Condições de saúde e nutrição de crianças indígenas e não-indígenas que vivem às margens do rio Solimões, Estado do Amazonas, Brasil. Cad Saúde Pública 2000; $16: 873$.

28. Frongillo Jr. EA, Onis M, Hanson KMP. Socioeconomic and demographic factors are associated with worldwide patterns of stunting and wasting of children. J Nutr 1997; 127:2302-9.

29. Engstrom EM, Anjos LA. Déficit estatural nas crianças brasileiras: relação com condições sócioambientais e estado nutricional materno. Cad Saúde Pública 1999; 15:559-67.

30. Drachler ML, Andersson MCS, Leite JCC, Marshall T, Aerts DRGC, Freitas PF, et al. Desigualdade social e outros determinantes da altura em crianças: uma análise multinível. Cad Saúde Pública 2003; 19:1815-25.

31. Victora CG, Vaughan JP, Kirkwood BR, Martines JC, Barcelos LB. Risck factors for malnutrition in Brazilian children: the role of social and environmental variables. Bull World Health Organ 1986; 64:299-309.

32. Carvalhaes MABL, Benício MHD. Capacidade materna de cuidar e desnutrição infantil. Rev Saúde Pública 2002; 36:188-97.

33. Immink MDC, Payongayong E. Risk analysis of poor health and growth failure of children in the central highlands of Guatemala. Soc Sci Med 1999; 48:997-1009.

34. Moore SR, Lima AAM, Conaway MR, Schorling JB, Soares AM, Guerrant RL. Early childhood diarrhoea and helminthiases associate with longterm linear growth faltering. Int J Epidemiol 2001; 30:1457-64.

35. Fontbonne A, Freese-de-Carvalho E, Acioli MD, Sá GA, Cesse EAP. Fatores de risco para poliparasitismo intestinal em uma comunidade indígena de Pernambuco, Brasil. Cad. Saúde Pública 2001; 17:367-73.

36. Organização Pan-Americana da Saúde/Organização Mundial da Saúde. 132ạ Sessão do Comitê Executivo. Etnicidade e saúde. http://newweb. www.paho.org/portuguese/gov/ce/ce132-16p.pdf (acessado em 30/Nov/2005).

Recebido em 12/Jan/2005

Versão final reapresentada em 30/Nov/2005 Aprovado em 26/Dez/2005 